\title{
In-hospital cardiac arrest and preceding National Early Warning Score (NEWS): A retrospective case-control study
}

\author{
Authors: Martin Spångfors, ${ }^{A}$ Mats Molt ${ }^{B}$ and Karin Samuelson ${ }^{C}$
}

\begin{abstract}
We aimed to describe and evaluate the National Early Warning Score (NEWS) in the $\mathbf{2 4}$ hours preceding an in-hospital cardiac arrest among general somatic ward patients.

The $\mathbf{2 4}$ hours preceding the in-hospital cardiac arrest were divided into four timespans and analysed by a medical record review of 127:254 matched case-control patients. The median NEWS ranged from $3(2-6)$ to 6 (3-9) points for cases vs 1 $(0-3)$ to $1(0-3)$ point for controls. The proportion of cases ranged from $23-45 \%$ at high risk vs $3-6 \%$ for controls. The NEWS high-risk category was associated with an increase of 3.17 (95\% confidence interval (CI) 1.66-6.04) to 4.43 (95\% CI 2.56-7.67) in odds of in-hospital cardiac arrest compared to the low-risk category.

NEWS, with its intuitive and for healthcare staff easy to interpret risk classification, is suitable for discriminating deteriorating patients with major deviating vital signs scoring high risk on NEWS.
\end{abstract}

KEYWORDS: National Early Warning Score, in-hospital cardiac arrest, critical care outreach, medical emergency team, early warning scores

\section{Introduction}

A majority (50-57\%) of the in-hospital cardiac arrests (IHCA) occur on hospital wards. ${ }^{1,2}$ The survival rate is low, and approximately $83 \%$ die within 30 -days and, in comparison, if IHCA occur in a cardiac catheterisation laboratory, the 30-day mortality rate is $37 \%{ }^{2}$ The research and guidelines for IHCA have predominantly focused on resuscitation and treatment when the event has already occurred and, despite these efforts, the survival after IHCA is low. ${ }^{2}$ To address this, the latest European Resuscitation Council (ERC) guidelines emphasised the need for preventing IHCA and, in order to take preventive steps, patients at risk of IHCA need to be identified early. ${ }^{3}$

Authors: ${ }^{\mathrm{A}}$ critical care registered nurse and PhD student, Lund University, Lund, Sweden and Hospital of Kristianstad, Kristianstad, Sweden; ${ }^{\mathrm{B}}$ affiliated researcher, Lund University, Lund, Sweden; Cassociate professor, Lund University, Lund, Sweden
Deviating vital signs have been known for decades to precede IHCA, and the National Early Warning Score (NEWS) is a tool designed to identify patients at risk of IHCA, unexpected death and of intensive care unit (ICU) admission within 24 hours. $^{4-9}$ NEWS has been shown to be superior to other early warning score instruments and is a recognised tool widely adopted in hospital settings across Europe. ${ }^{10-15}$ NEWS classifies the clinical risk for critical illness into low, medium and high risk, providing a clinically useful way to discriminate patients at risk of suffering an IHCA. In a clinical setting it might be difficult for the healthcare staff to relate the total score to the risk of serious adverse events like IHCA whereas the NEWS risk classification offers a more intuitive clinical risk stratification. However, previous studies have focused on the total score and shown that IHCA, compared to unexpected death and ICU admission, appears to be the most complicated adverse event for NEWS to discriminate. When using the total score measured some time during the 24 preceding hours, the commonly used area under the curve (AUC) ranged from 0.72-0.78 for IHCA compared to 0.86-0.91 for unexpected death and $0.86-0.86$ for ICU admission. $9,10,12$

Further evaluation of NEWS in this area is needed even though this previous research indicates that NEWS is a promising tool for the detection of IHCA. Knowledge about NEWS when using the clinical risk classification groups (low, medium and high) might contribute to the prevention of IHCA, providing opportunities to intervene and prevent unnecessary suffering.

Moreover, the degree and timing of physiological instability in the 24 hours preceding IHCA have been shown to differ between studies. 4,5 Instability for as long as 8-24 hours before the IHCA has been reported but none of these studies reflect the dynamics of NEWS. 4,5

The aim of the study was to describe NEWS in different timespans in the 24 hours preceding IHCA and to evaluate the discriminative ability of NEWS among general somatic ward patients, using the clinical risk classification.

\section{Materials and methods \\ Study design and setting}

This is a retrospective multicentre medical record review study, using a 1:2 matched case-control design. Three emergency hospitals in Sweden, comprising one university hospital with 997 adult beds and two regional hospitals with 304 and 246 adult beds participated (catchment area population of 1.3 million citizens). 


\section{Patients}

All patients, $\geq 18$ years of age, admitted for at least 24 hours, suffering an IHCA on a general somatic hospital ward from 01 January 2016 to 31 December 2017 were reviewed for eligibility. Patients suffering an IHCA in the ICU, cardiac high-dependency unit, cardiac catheterisation laboratory, operating theatre, postoperative recovery unit or in the emergency department were not considered for inclusion. Further, patients with chronic obstructive pulmonary disease (COPD) were excluded because their oxygen saturation should be judged individually depending on their habitual state. ${ }^{8}$ Patients without any NEWS measurements during the studied period were also excluded. The included patients with IHCA (cases) were matched with controls without IHCA in a 1:2 ratio by the same admission year, hospital, ward, sex, age \pm 5 years, primary admission diagnosis or admission diagnosis chapter according to the International Classification of Diseases 10th revision (ICD-10). ${ }^{16}$

\section{Definitions}

NEWS is calculated from measures of respiratory rate, oxygen saturations, body temperature, systolic blood pressure, heart rate and level of consciousness which are rated from $0-3$, correlating with their divergence from the expected normal values, and summed. Supplemental oxygen increases the score by 2 points. ${ }^{8}$ According to guidelines, the NEWS clinical risk scale was calculated as follows; low risk $=0-4$ points, medium risk $=5-6$ points or 3 points in one parameter and high risk $=\geq 7$ points. ${ }^{8}$

Comorbidity was assessed by the Charlson comorbidity index (CCI) and the age-adjusted Charlson comorbidity index (ACCI). ${ }^{17}$ Further, ACCI was categorised into minimal, low, moderate or severe 'burden of age-combined comorbidities' if the ACCI was $0-2$ points, $3-5$ points, $6-7$ points or 8 points and higher, respectively. ${ }^{18}$

The hospitals in the study participate in the Swedish Cardiac Arrest Registry and their definition of IHCA was used, ie a patient who is unresponsive with apnoea or agonal, gasping respiration where cardiopulmonary resuscitation and/or defibrillation was initiated. ${ }^{19}$

\section{Data collection}

Cases were identified in the hospitals' documentation systems and cardiac arrest records. The following data were collected by the researchers and stored in an electronic database: hospital, ward, date of admission, date of IHCA, age, sex, primary diagnosis according to the International Statistical Classification of Diseases and Related Health Problems 10th revision (ICD-10), comorbidity, vital signs, NEWS-parameters and hospital mortality. After inclusion, the hospitals' electronic medical records were searched for matching controls. When the data of both cases and controls were collected, the electronic database was searched both automated and manually for illogical values by one of the authors. For calculation of NEWS, at least 4 of the 7 parameters needed to be registered at the same time and the missing parameters had to be documented in another NEWS measurement during the 24 hours, otherwise the NEWS was categorised as missing. The last measurement was carried forward manually to replace the missing value.

\section{Ethics}

This study was approved by the regional Research Ethical Review Board in Lund, Sweden which waived the need for informed consent (Dnr 2016/940). The study is reported in accordance with the strengthening the reporting of observational studies in epidemiology (STROBE) statement. ${ }^{20}$

\section{Statistical analysis}

An a priori sample size calculation showed that 100 cases and 200 controls would generate a power of $>80 \%$ to detect an odds ratio of 2.0 with an $\alpha$-level of 0.05 . Categorical and nonparametric data are presented with median scores (25-75 percentiles). The Mann-Whitney $U$-test and $\chi^{2}$ test were used to test for differences between cases and excluded. We chose to divide the data into four different 6 -hour timespans (24-18 hours, 18-12 hours, 12-6 hours and $6-0$ hours) preceding the IHCA with inspiration from a report of the National Confidential Enquiry into Patient Outcome and Death. ${ }^{5}$ In case of multiple NEWS measurements within each timespan, the highest NEWS value was chosen. For control patients, the highest NEWS value during the 24 studied hours was chosen as their study period was chosen arbitrarily ie without a fixed time of event. The Friedman test was used to test for differences between cases and controls. The Wilcoxon signed rank test was used to test for differences between cases in different timespans.

Conditional logistic regression analysis was used with high, medium and low risk as independent variables for prediction of IHCA and presented as odds ratio (OR) with $95 \%$ confidence interval (CI). The CCI, ACCI, categorised ACCI, sex, medical affiliation and the metric variable age were tested as covariates.

The ability of the NEWS risk classification to discriminate IHCA was performed by AUC. Statistical significance was set at $p<0.05$. All analyses were performed with IBM SPSS, v25.

\section{Results}

\section{Baseline characteristics}

A total of 127 patients suffering an IHCA (cases) was included (Fig 1). Median age of the cases was $73(62-80)$ years, $76(60 \%)$ were male and 80 (63\%) were medical patients (Table 1). When including the 254 control patients there was a total of 970 NEWS measurements and missing data occurred in 203 (21\%) of these. The most common missing NEWS parameter was temperature, in $178(18 \%)$ NEWS measurements, followed by supplemental oxygen, in 10 (1\%) NEWS measurements. Twenty-six of the 970

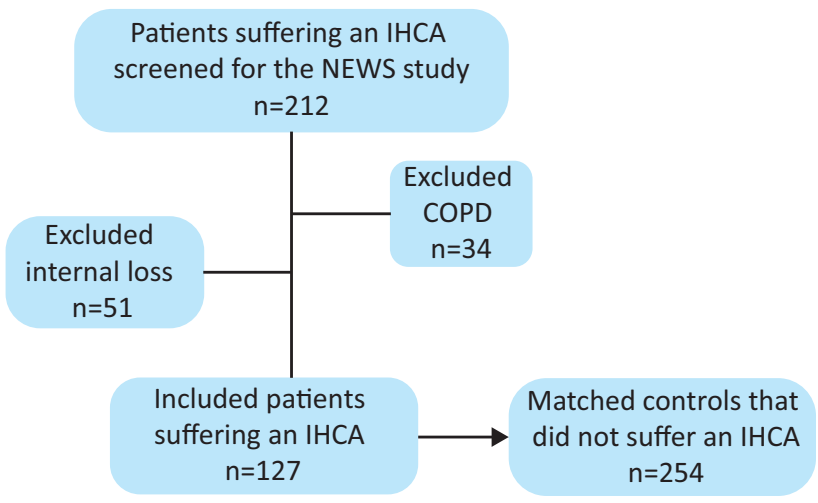

Fig 1. Flow diagram of general ward patients included. $C O P D=$ chronic obstructive pulmonary disease; IHCA = in-hospital cardiac arrest; NEWS = National Early Warning Score. 
Table 1. Characteristics of the included and excluded general ward patients

\begin{tabular}{|c|c|c|c|c|}
\hline Variable & $\begin{array}{l}\text { Included with IHCA } \\
\text { (cases), } n=127\end{array}$ & $\begin{array}{l}\text { Included without IHCA } \\
\text { (controls), } n=254\end{array}$ & $\begin{array}{l}\text { Excluded with } \\
\text { IHCA, } n=85\end{array}$ & $\begin{array}{l}\text { Included IHCA vs } \\
\text { excluded IHCA, p-value }\end{array}$ \\
\hline Age, years (range) & $73(62-80)$ & $73(64-80)$ & $74(66-82)$ & 0.27 \\
\hline Sex, male, n (\%) & $76(60)$ & $152(60)$ & $52(61)$ & 0.85 \\
\hline Clinical affiliation: & & & & 0.54 \\
\hline Medicine, n ( \%) & $80(63)$ & $160(63)$ & $50(59)$ & \\
\hline Surgery, n (\%) & $47(37)$ & $94(37)$ & $35(41)$ & \\
\hline $\begin{array}{l}\text { Main reasons for admission according } \\
\text { to the ICD10 codes: }\end{array}$ & & & & 0.06 \\
\hline Neoplastic disease C00-D48, n (\%) & $11(9)$ & $22(9)$ & $7(8)$ & \\
\hline Circulatory system I00-99, n (\% ) & $15(12)$ & $30(12)$ & $11(13)$ & \\
\hline Respiratory system J00-99, n (\%) & $11(9)$ & $22(9)$ & $19(22)$ & \\
\hline Injury/trauma S00-T98, n (\%) & $11(9)$ & $22(9)$ & $6(7)$ & \\
\hline Gastrointestinal system K00-93, n (\%) & $10(8)$ & $20(8)$ & $5(6)$ & \\
\hline Musculoskeletal system M00-99, n (\%) & $7(6)$ & $14(6)$ & $0(0)$ & \\
\hline Infectious diseases A00-B99, n (\%) & $10(8)$ & $20(8)$ & $4(5)$ & \\
\hline $\begin{array}{l}\text { Symptoms, signs and abnormal } \\
\text { clinical and laboratory findings not } \\
\text { elsewhere classified R00-99, n (\%) }\end{array}$ & $37(29)$ & $74(29)$ & $19(22)$ & \\
\hline $\begin{array}{l}\text { Hospital length of stay when IHCA } \\
\text { occurred, median days (range) }\end{array}$ & $3(2-8)$ & $\mathrm{n} / \mathrm{a}$ & $5(2-9)$ & 0.77 \\
\hline In-hospital mortality, n (\%) & $97(76)$ & $6(2)$ & $65(77)$ & 0.87 \\
\hline $\begin{array}{l}\text { Age-adjusted Charlson comorbidity } \\
\text { index, points (range) }\end{array}$ & $3(5-7)$ & $3(4-6)$ & $4(5-6)$ & 0.74 \\
\hline \multicolumn{5}{|l|}{$\begin{array}{l}\text { Categorised burden of age-adjusted } \\
\text { Charlson comorbidity index: }\end{array}$} \\
\hline Minimal 0-2 points, $\mathrm{n}(\%)$ & $21(16)$ & $55(22)$ & $\mathrm{n} / \mathrm{a}$ & \\
\hline Low 3-5 points, n ( \%) & $58(46)$ & $108(42)$ & $\mathrm{n} / \mathrm{a}$ & \\
\hline Moderate 6-7 points, n (\%) & $28(22)$ & $60(24)$ & $\mathrm{n} / \mathrm{a}$ & \\
\hline Severe $\geq 8$ points, $\mathrm{n}(\%)$ & $20(16)$ & $31(12)$ & $\mathrm{n} / \mathrm{a}$ & \\
\hline
\end{tabular}

NEWS measurements were upgraded to medium risk due to a score of 3 in a single parameter. Of the NEWS measurements, 226 were excluded due to multiple NEWS measurements within its timespan, leaving 744 for further analysis.

\section{NEWS in different timespans}

In the timespan 24-18 hours before IHCA, 56 cases had a NEWS assessment and the median NEWS was 3 (2-6) points vs $1(0-3)$ point for controls $(p<0.001)$. Among cases, $23 \%$ were at high risk and $16 \%$ at medium risk vs $3 \%$ and $7 \%$, respectively, among the controls $(p<0.001$; Table 2). Medium or high risk on NEWS was associated with an increase in the odds of 2.47 (95\% CI 1.18-5.17, $p=0.016)$ and 3.17 (95\% CI 1.66-6.4, p<0.001) for IHCA, respectively, compared to low risk. AUC for the NEWS risk classification in discriminating IHCA was 0.58 (95\% CI 0.49-0.67, $p=0.087$; Table 3).

In the timespan 18-12 hours before IHCA, 63 cases had a NEWS assessment and the median NEWS was 4 (2-6) points vs 1 (0-3) point for controls $(p<0.001)$. Among cases, $18 \%$ were at high risk and $30 \%$ at medium risk vs the controls, $3 \%$ and $12 \%$, respectively $(p<0.001$; Table 2). Medium or high risk on NEWS was associated with an increase in the odds of 2.33 (95\% CI 1.32-4.11, $p=0.003)$ and 3.57 (95\% CI 1.79-7.10, p<0.001) for IHCA, respectively, compared to low risk. AUC for the NEWS risk classification in discriminating IHCA was 0.61 (95\% CI 0.52-0.69, $p=0.018$; Table 3).

In the timespan 12-6 hours before IHCA, 67 cases had a NEWS assessment and the median NEWS was 4 (2-6) points vs 1 (1-3) point for controls $(p<0.001)$. Among cases, $24 \%$ were at high risk and $22 \%$ at medium risk vs the controls $3 \%$ and $16 \%$, respectively ( $p<0.001$; Table 2). Medium or high risk on the NEWS was associated with an increase in the odds of 1.59 (95\% CI 0.87-2.92, $p=0.131)$ and 3.69 (CI 2.04-6.67, p<0.001) for IHCA, respectively, compared to low risk. AUC for the NEWS risk classification in discriminating IHCA was 0.59 (95\% CI 0.51-0.67, $p=0.041$; Table 3). In the timespan 6-0 hours before IHCA, 62 cases had a NEWS assessment and the median NEWS was 6 (3-9) points for cases 
Table 2. The NEWS risk classification in the different timespans

\begin{tabular}{|c|c|c|c|}
\hline Timespans & $\begin{array}{l}\text { Patients with } \\
\text { IHCA (cases) }\end{array}$ & $\begin{array}{l}\text { Patients without } \\
\text { IHCA (controls) }\end{array}$ & $\begin{array}{l}\text { Cases vs controls, } \\
\text { p-value }\end{array}$ \\
\hline NEWS risk classification 24-18 hours before IHCA: & $n=56$ & $\mathrm{n}=112$ & $<0.001$ \\
\hline Low, $\mathrm{n}(\%)$ & $34(61)$ & $101(90)$ & \\
\hline Medium, n (\%) & $9(16)$ & $8(7)$ & \\
\hline High, n (\%) & $13(23)$ & $3(3)$ & \\
\hline NEWS risk classification $18-12$ hours before IHCA: & $n=63$ & $n=126$ & $<0.001$ \\
\hline Low, n (\%) & $33(52)$ & $107(85)$ & \\
\hline Medium, n (\%) & $19(30)$ & $15(12)$ & \\
\hline High, n (\%) & $11(18)$ & $4(3)$ & \\
\hline p-value between timespan $24-18$ vs $18-12$ hours & 0.819 & $\mathrm{n} / \mathrm{a}$ & \\
\hline NEWS risk classification 12-6 hours before IHCA: & $n=67$ & $n=134$ & $<0.001$ \\
\hline Low, $\mathrm{n}(\%)$ & $36(54)$ & $109(81)$ & \\
\hline Medium, n (\%) & $15(22)$ & $21(16)$ & \\
\hline High, n (\%) & $16(24)$ & $4(3)$ & \\
\hline p-value between timespan $18-12$ vs $12-6$ hours & 0.658 & $\mathrm{n} / \mathrm{a}$ & \\
\hline NEWS risk classification 6-0 hours before IHCA: & $n=62$ & $n=124$ & $<0.001$ \\
\hline Low, n (\%) & $24(39)$ & $100(81)$ & \\
\hline Medium, n (\%) & $10(16)$ & $16(13)$ & \\
\hline High, n (\%) & $28(45)$ & $8(6)$ & \\
\hline p-value between timespan $12-6$ vs $6-0$ hours & 0.048 & $\mathrm{n} / \mathrm{a}$ & \\
\hline
\end{tabular}

and $1(0-3)$ point for controls $(p<0.001)$. Among cases, $45 \%$ were at high risk and $16 \%$ at medium risk vs the controls $6 \%$ and $13 \%$, respectively, $(p<0.001$; Table 2$)$. Medium or high risk on the NEWS was associated with an increase in the odds of $1.63(95 \%$ CI $0.78-3.42, p=0.195)$ and 4.43 (95\% CI 2.56-7.67, $p<0.001)$ for IHCA, respectively, compared to low risk. AUC for the NEWS risk classification in discriminating IHCA was 0.64 (95\% CI 0.56-0.72, $\mathrm{p}=0.002$; Table 3).

The CCI, ACCI, categorised ACCI, sex, medical affiliation and the metric variable age were tested as covariates in the different timespans but not found to be significant.

When testing the distribution of the NEWS risk categories among cases in different timespans, a difference was found between 0-6 hours and $6-12$ hours before IHCA ( $p=0.04$; Table 2$)$.

\section{Discussion}

The results in our study suggest a process of clinical deterioration in patients suffering an IHCA, with the timespan $6-0$ hours being the most favourable for NEWS to identify patients at risk. In all timespans, $18-24 \%$ of cases were classified as high risk whereas in the timespan 6-0 hours the percentage almost doubled. The corresponding proportion among controls was $3-6 \%$ during all timespans. Further, there was a more than threefold increase in odds of IHCA in the high-risk group compared to the low-risk group during all timespans, indicating that a large proportion of patients suffering an IHCA can be detected up to 24 hours prior to the incident.

Medium risk seems to be the most challenging group to differentiate against since the difference in proportion of patients

Table 3. Conditional logistic regression analysis for IHCA on the NEWS risk classification

\begin{tabular}{|c|c|c|c|c|}
\hline $\begin{array}{l}\text { NEWS risk classification } \\
\text { compared to low risk }\end{array}$ & $\begin{array}{l}24-18 \text { hours before } \\
\text { IHCA }\end{array}$ & $\begin{array}{l}\text { 18-12 hours before } \\
\text { IHCA }\end{array}$ & $\begin{array}{l}\text { 12-6 hours before } \\
\text { IHCA }\end{array}$ & $\begin{array}{l}6-0 \text { hours before } \\
\text { IHCA }\end{array}$ \\
\hline $\begin{array}{l}\text { Medium, OR (95\% CI, } \\
\text { p-value) }\end{array}$ & $\begin{array}{l}2.47 \\
(1.18-5.17, p=0.016)\end{array}$ & $\begin{array}{l}2.33 \\
(1.32-4.11, p=0.003)\end{array}$ & $\begin{array}{l}1.59 \\
(0.87-2.92, p=0.131)\end{array}$ & $\begin{array}{l}1.63 \\
(0.78-3.42, p=0.195)\end{array}$ \\
\hline $\begin{array}{l}\text { High, OR (95\% CI, } \\
\text { p-value) }\end{array}$ & $\begin{array}{l}3.17 \\
(1.66-6.04, p<0.001)\end{array}$ & $\begin{array}{l}3.57 \\
(1.79-7.10, p<0.001)\end{array}$ & $\begin{array}{l}3.69 \\
(2.04-6.67, p<0.001)\end{array}$ & $\begin{array}{l}4.43 \\
(2.56-7.67, p<0.001)\end{array}$ \\
\hline AUC (95\% CI, p-value) & $\begin{array}{l}0.58 \\
(0.49-0.67, p=0.087)\end{array}$ & $\begin{array}{l}0.61 \\
(0.52-0.69, p=0.018)\end{array}$ & $\begin{array}{l}0.59 \\
(0.51-0.67, p=0.041)\end{array}$ & $\begin{array}{l}0.64 \\
(0.56-0.72, p=0.002)\end{array}$ \\
\hline
\end{tabular}

$\mathrm{AUC}=$ area under the curve; $\mathrm{CI}=$ confidence interval; $\mathrm{IHCA}=$ in-hospital cardiac arrest; NEWS = National Early Warning Score; OR = crude odds ratio. 
suffering an IHCA compared to others was not particularly large, and it might be difficult for a ward-based physician not skilled in the assessment of acutely ill patients to assess these patients. In the revised NEWS2 outline clinical response scale, a clinician or team with competence in the assessment and treatment of acutely ill patients is recommended at this level. ${ }^{21}$ It seems that this might be a crucial step to find patients truly at risk of IHCA in this risk category.

The overall discriminative ability of NEWS as tested by the AUC was poor. The greatest discriminative ability was seen in the timespan 6-0 hours before IHCA but it was still considered low (0.64), indicating that many patients might be missed. Our study showed that a large proportion of patients suffering an IHCA show minor deviating vital signs in the preceding $24-6$ hours, thus making it more difficult for NEWS to discriminate in these timespans. Previous studies showed greater AUC values for unexpected death and ICU-admission than for IHCA and support these findings. ${ }^{9,10,12}$

In a study by Nolan et al, IHCA occurred in a median of 48 hours after admittance to the hospital and, in our study, IHCA occurred in a median of 72 hours after admittance among patients being admitted for more than 24 hours to the hospital, signalling that there might be a window of opportunity to detect deterioration in many patients. ${ }^{1}$ Survival after IHCA has previously been shown to be associated with comorbidity as shown in the study by Roberts et $a l^{18}$ However, as in the study by Tirkkonen et al, where patients had been reviewed by the rapid response team prior to IHCA, the CCI was not found to be a significant confounder associated with IHCA in our study. ${ }^{22}$ It seems that comorbidity is not associated with the incidence of an IHCA. However, survival after IHCA seems to be associated with comorbidity as shown in the study by Roberts et al. ${ }^{18}$

Approximately $39 \%$ of patients suffering an IHCA were classified as low risk in the timespan 6-0 hours. However, none of the patients suffering an IHCA had a NEWS of 0 points, which might warrant a new risk category of 'Low-low' or 'Minimal' where those with a NEWS of 0 points are placed. This might increase the discriminative ability of patients at risk and increase hospital staff's awareness of these patients. Further, our results raise the question whether intermittent evaluation of a patient's vital signs is appropriate or if continuous vital signs monitoring should be used on all patients with deviating vital signs.

\section{Limitations and strengths}

Missing recordings of vital signs is a well-known problem in healthcare settings. ${ }^{5,22-26}$ In our study, missing data occurred, mostly concerning temperature and, in some cases, supplemental oxygen. We tried mitigating the effects of missing variables by inputting the last recorded value in the parameter and we do not suspect this has introduced any significant bias. Further, temperature has previously been shown not to be a predictor of IHCA and there was just $1 \%$ missing in supplemental oxygen, which is deemed non-significant to the results. ${ }^{27}$

Another limitation was the exclusion of patients; those with COPD which were done because their oxygen saturation should be judged individually depending on their habitual state and those suffering an IHCA without having a documented NEWS in the preceding 24 hours. No major differences were found comparing patient characteristics between excluded and included patients, but a tendency towards a difference in main reasons for admission as categorised by the ICD-10, which was probably due to us deciding to exclude all patients with COPD.

We chose to use the highest NEWS in the different timespans, if multiple NEWS were available, this might be a limitation if it is not the closest one to the IHCA. Since the timespan 6-0 hours before IHCA was the most important for detecting deterioration in our study we considered performing a subgroup analysis. However, there were 20 patients with multiple measurements $0-6$ hours preceding IHCA. Of these, 18 patients had the highest NEWS closest to the IHCA and since only two patients did not, we did not perform a subgroup analysis.

Furthermore, the observational retrospective study design only allowed associations to be drawn and not causality, and we thus tried to clarify our results by adjusting for possible confounders in age, sex, comorbidity and medical affiliation.

A strength in our study is the multicentre approach where both a large university hospital and smaller community hospitals were included as this might increase the generalisability.

\section{Conclusion}

The proportion of patients classified as high risk almost doubled between $12-6$ and $6-0$ hours before the IHCA, indicating a dynamic process of deterioration.

NEWS high risk was associated with a more than threefold odds of IHCA compared to low risk during the preceding 24 hours.

NEWS, with its intuitive and for healthcare staff easy to interpret risk classification, is thus suitable for discriminating deteriorating patients with major deviating vital signs scoring high risk on NEWS.

\section{References}

1 Nolan JP, Soar J, Smith GB et al. Incidence and outcome of inhospital cardiac arrest in the United Kingdom National Cardiac Arrest Audit. Resuscitation 2014;85:987-92.

2 Herlitz J. [Swedish cardiopulmonary register: Annual report 2016]. Stockholm: Svenska rådet för hjärt-lungräddning, 2016. https:// registercentrum.blob.core.windows.net/shlrsjh/r/-rsrapport-2016SypN5EYqb.pdf

3 Soar J, Nolan JP, Bottiger BW et al. European Resuscitation Council Guidelines for Resuscitation 2015: Section 3. Adult advanced life support. Resuscitation 2015;95:100-47.

4 Schein RMH, Hazday N, Pena M et al. Clinical antecedents to inhospital cardiopulmonary arrest. Chest 1990;98:1388-92.

5 Findlay GP, Shotton H, Kelly K, Mason M. Time to intervene? A review of patients who underwent cardiopulmonary resuscitation as a result of an in-hospital cardiorespiratory arrest. London: National Confidential Enquiry into Patient Outcome and Death, 2012. www.ncepod.org.uk/2012report1/downloads/CAP_fullreport. pdf [Accessed 16 March 2019].

6 Kause J, Smith G, Prytherch D et al. A comparison of antecedents to cardiac arrests, deaths and emergency intensive care admissions in Australia and New Zealand, and the United Kingdom-the ACADEMIA study. Resuscitation 2004;62:275-82.

7 Andersen LW, Kim WY, Chase M et al. The prevalence and significance of abnormal vital signs prior to in-hospital cardiac arrest. Resuscitation 2016;98:112-7.

8 Royal College of Physicians. National Early Warning Score (NEWS): Standardising the assessment of acute illness severity in the NHS. Report of a working party. London: RCP, 2012. www.rcplondon. ac.uk/projects/outputs/national-early-warning-score-news-2 [Accessed 16 March 2019]. 
9 Smith GB, Prytherch DR, Meredith P et al. The ability of the National Early Warning Score (NEWS) to discriminate patients at risk of early cardiac arrest, unanticipated intensive care unit admission, and death. Resuscitation 2013;84:465-70.

10 Kovacs C, Jarvis SW, Prytherch DR et al. Comparison of the National Early Warning Score in non-elective medical and surgical patients. Br J Surg 2016;103:1385-93.

11 Petersen JA, Antonsen K and Rasmussen LS. Frequency of early warning score assessment and clinical deterioration in hospitalized patients: A randomized trial. Resuscitation 2016;101:91-6.

12 Smith GB, Prytherch DR, Jarvis $S$ et al. Comparison of the ability of the physiologic components of medical emergency team criteria and the UK National Early Warning Score to discriminate patients at risk of a range of adverse clinical outcomes. Crit Care Med 2016;44:2171-81.

13 Spangfors M, Arvidsson L, Karlsson V et al. The National Early Warning Score: Translation, testing and prediction in a Swedish setting. Intensive Crit Care Nurs 2016;37:62-7.

14 Tirkkonen J, Olkkola KT, Huhtala $\mathrm{H}$ et al. Medical emergency team activation: performance of conventional dichotomised criteria versus national early warning score. Acta Anaesthesiol Scand 2014;58:411-9.

15 Spagnolli W, Rigoni M, Torri E et al. Application of the National Early Warning Score (NEWS) as a stratification tool on admission in an Italian acute medical ward: A perspective study. Int J Clin Pract 2017;71:e12934.

16 World Health Organization. International Statistical Classification of Diseases and Related Health Problems 10th Revision (ICD-10). Geneva: WHO, 2016. https://icd.who.int/browse10/2016/en [Accessed 16 March 2019].

17 Charlson M ST, Peterson J, Gold J. Validation of a combined comorbidity index. J Clin Epidemiol 1994;47:1245-51.

18 Roberts D, Djarv T. Preceding national early warnings scores among in-hospital cardiac arrests and their impact on survival. Am J Emerg Med 2017:35:1601-6.
19 Herlitz ]. [Swedish cardiopulmonary register: Annual report 2017]. Stockholm: Svenska rådet för hjärt-lungräddning, 2017. https:// registercentrum.blob.core.windows.net/shlrsjh/r/-rsrapport-2017 Sy1wvd12Z.pdf

20 Vandenbroucke JP, von Elm E, Altman DG et al. Strengthening the Reporting of Observational Studies in Epidemiology (STROBE): explanation and elaboration. Epidemiology 2007;18:805-35.

21 Royal College of Physicians. National Early Warning Score (NEWS) 2: Standardising the assessment of acute-illness severity in the NHS. Updated report of a working party. London: RCP, 2017. www.rcplondon.ac.uk/projects/outputs/national-early-warningscore-news-2 [Accessed 16 March 2019].

22 Tirkkonen J, Huhtala H, Hoppu S. In-hospital cardiac arrest after a rapid response team review: A matched case-control study. Resuscitation 2018;126:98-103.

23 Pedersen NE, Rasmussen LS, Petersen JA et al. A critical assessment of early warning score records in 168,000 patients. J Clin Monit Comput 2018;32:109-16.

24 Hands C, Reid E, Meredith P et al. Patterns in the recording of vital signs and early warning scores: compliance with a clinical escalation protocol. BMJ Qual Saf 2013;22:719-26.

25 Kolic I, Crane S, McCartney S et al. Factors affecting response to national early warning score (NEWS). Resuscitation 2015;90 :85-90.

26 Prytherch DR, Smith GB, Schmidt P et al. Calculating early warning scores - a classroom comparison of pen and paper and hand-held computer methods. Resuscitation 2006;70:173-8.

27 Churpek MM, Yuen TC, Huber MT et al. Predicting cardiac arrest on the wards: a nested case-control study. Chest 2012;141:1170-6.

Address for correspondence: Martin Spångfors, Department of Anaesthesiology and Intensive Care, Hospital of Kristianstad, 29189 Kristianstad, Region Skåne, Sweden. Email: martin.spangfors@skane.se 